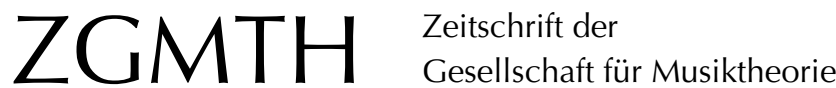

Fladt, Hartmut (2011): Matthias Schlothfeldt, Komponieren im Unterricht (= FolkwangStudien 9, hg. von Stefan Orgass und Horst Weber), Hildesheim/Zürich/New York: Olms 2009. ZGMTH 8/1, 197-198. https://doi.org/10.31751/584

(C) 2011 Hartmut Fladt

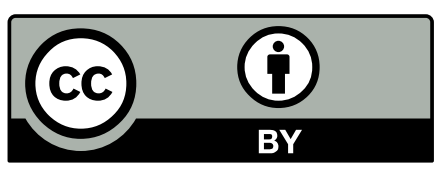

Dieser Text erscheint im Open Access und ist lizenziert unter einer Creative Commons Namensnennung 4.0 International Lizenz.

This is an open access article licensed under a Creative Commons Attribution 4.0 International License.

veröffentlicht / first published: 14/06/2011

zuletzt geändert / last updated: 21/04/2011 


\section{Matthias Schlothfeldt, Komponieren im Unterricht (= FolkwangStudien 9, hg. von Stefan Orgass und Horst Weber), Hildesheim/Zürich/New York: Olms 2009}

'Schüler komponieren`, 'Jugend komponierts - das sind, auch außerhalb der Schulen, sowohl institutionell als auch durch ifreie Kurse, Wettbewerbe etc. fest etablierte Einrichtungen, die auch schon eine erhebliche Anzahl von Publikationen hervorgebracht haben, seien es nun Erfahrungsberichte, Konzeptionen, kompositorische Maßnahmen, pädagogische Reflexionen.

Die Schwerpunktbildungen der Verantwortlichen wechseln: lehrende und publizierende Komponisten haben in der Regel andere Zielsetzungen als Musiktheoretiker oder Pädagogen. Matthias Schlothfeldt (als Komponist, Musiktheoretiker und Musikpädagoge zugleich) versucht nun eine Synthese der bisweilen divergierenden Ansätze, die sowohl praxisorientiert als auch theoretischreflektierend ist. Sie vermeidet ein abstraktes >Pädagogisieren TTheoretisieren` oder leeres «ünstlern`.

In Lehr- und Prüfungsplänen fest verankert, doch immer wieder Rat- und Planlosigkeit verbreitend: das Komponieren in der Schule selbst, im Klassenverband und in Kursen. Das ist bisher primär eine Domäne der Pädagogik, wobei die Lehrer/Studienrats-Ausbildung da vielfache Defizite aufweist, die Matthias Schlothfeldt benennt (und zu deren Überwindung er mit diesem Buch von über 300 Seiten ebenfalls beiträgt). Konsequenzen aus den Erfahrungen des Pragmatikers und des Pädagogen stehen hier in einem permanent produktiven Spannungs-Verhältnis zu den Maximen und Wünschen des Wissenschaftlers und des Komponisten.

Die Position gerade der Musiktheorie zwischen historischer und systematischer
Wissenschaftlichkeit, zwischen analytischer und handwerklicher Kompetenzvermittlung, kompositorischer/improvisatorischer Kreativität und pädagogischer shandlungsorientierter Reflexion` wird auf eindrucksvolle Weise als Chance begriffen: 'Theorie seröffnet so ein Potential, das sich in den einzelnen sSparten allein nie ergeben würde.

Über Konzeptionelles und einige Details sei jetzt genauer Auskunft gegeben. Ein Bündel von quantitativ umfassenden und zugleich qualitativ substantiellen Leitfragen prägt die drei Haupt-Abschnitte des Buchs:

- Musiktheorie, Komposition, Didaktik (mit umfassender sPraxisı-Orientierung und Nachdenken über die Funktionen der Improvisation)

- Kompositionspädagogische Erfahrungen und Vorschläge (Beispiele, Pragmatik der Rahmenbedingungen, Rolle des Lehrers, Analysen, inhaltliche und qualitative Kriterien; mit sSchülerbefragungen`)

- Entwurf einer Didaktik der Musiktheorie (Analyse, Tonsatz, Improvisation, Musik und Sprache)

Wie schon der flüchtige Blick zeigt, gibt es hier auch für die Musiktheorie an unseren Hochschulen umfassende Anregungen, besonders - aber eben nicht nur - für die Lehramts-Studiengänge. Wie viel `Neue Musikı ist nötig, welche Erfahrungen benötigen Schülerinnen und Schüler, um kreativ-Eigenes aus der Erfahrung von umfassend Gegenwärtigem fruchtbar machen zu können? Schließen sich Kreativität und traditioneller Tonsatz aus? Wie kann die postmoderne Konzeption von sMu- 
sik über Musikı gerade bei Grenzüberschreitungen von Traditionellem und Neuem für die Schule in Anspruch genommen werden?

Wenn ein Hauptanliegen so formuliert wird, dass die Schülerinnen und Schüler bei den musikalischen Erfahrungen, bei den sMusik-Sprachen abgeholt werden, die ihnen vertraut sind, dann ist es unvermeidbar, dass auch Blues, Rock- und Pop-Musik integriert werden. Und: es geschieht (z. B. Beatles, Red Hot Chili Peppers); nach meinen Erfahrungen könnte es aber - oder müsste sogar - noch mehr sein. Auch in dieser Musik gibt es unendlich viel Experimentelles und von Neuer Musik substantiell Inspiriertes; das könnte analytisch verdeutlicht werden und Anregungen für eigene Grenzüberschreitungen liefern.

Das Plädoyer für die noten-schriftliche $\mathrm{Fi}$ xierung von kompositorischen Resultaten, gegen die Perpetuierung von musikalischem Analphabetismus ist leidenschaftlich. Und auch zumindest das musiktheoretische Basiswissen mit grundlegenden Termini wird eingefordert, um die verbreitete, oft dumpfe Sprachlosigkeit zu konterkarieren.
Das Unterkapitel über sschöne Stellen und den produktiven Umgang mit ihnen - das ist selbst eine der sschönen Stellen des Buchs, die zu lesen hohes Vergnügen bereitet.

Kritische Anmerkungen gibt es unter anderem zum (weit verbreiteten) mechanischen Anwenden der Zwölftontechnik: das abstrakte Abzählen schickt den musikalischen Sinn zum Teufel. Und auch das, was an musikalisch-unmusikalischen `Regeln « der Dodekaphonie in manchen Schulbüchern (aber leider auch in manchen Theorie-Büchern) zu lesen ist, wird kritisch moniert.

Die Fülle der Erfahrungen und der daraus resultierenden Anregungen, das Reflexionsniveau und die Stärken der systematischen Verknüpfungen lassen dieses Buch im Kontext der bisherigen Publikationen mit vergleichbarem Anliegen auf fast beängstigende Weise herausragen. Nicht nur die Schülerinnen und Schüler und die Studierenden werden, bei allem Spielerischen und bei allem Spaß, ernst genommen, sondern auch wir Mit-Lehrenden.

Hartmut Fladt 